\title{
Diversity and abundance of birds in Reiek Biodiversity Spot, Mizoram, northeastern India
}

\author{
C. Lalruatkimi ${ }^{1}$, S.S. Sundaravel ${ }^{1}$, Lalawmawia Sailo ${ }^{2}$, Betsy Zodinpuii ${ }^{2}$, Hmar Lalthanzara $^{2 *}$ \\ ${ }^{1}$ Department of Ecology and Environmental Sciences, Pondicherry University, Puducherry 605014, India \\ ${ }^{2}$ Department of Zoology, Pachhunga University College, Aizawl 796001, India
}

\begin{abstract}
Birds are one of the best-known classes of living organisms, they are important bioindicators of an ecosystem. This study was undertaken to determine the diversity and abundance of birds in Reiek Biodiversity Spot, Mizoram, northeastern India. An intensive study was carried out by line transect method. A total of 117 species of birds belonging to 37 families and 10 orders were recorded and the Shannon-Wiener Diversity index was calculated $\left(H^{\prime}=3.85\right)$. Family Muscicapidae dominated the area comprising of 16 species, followed by Timaliidae with 6 species. Among all the species recorded, Alcippe nipalensis has the highest relative abundance (13.35\%), followed by Staphida castaniceps (Striated Yuhina) and Zosterops palpebrosus (Oriental White Eye) with $8.6007 \%$ and $6.03337 \%$ each. Family-wise relative abundance revealed that Pycnonotidae has the highest relative abundance (17.45\%), followed by Sylviidae (15.91\%) and Timaliidae (13.35\%). The area supports a rich and diverse avian community, therefore, recommended better management of the entire landscape.
\end{abstract}

Keywords: Abundance, birds, diversity, forest, Reiek Biodiversity Spot.

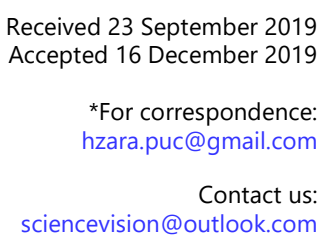

sciencevision@outlook.com

\section{Introduction}

Birds are one of the best-known classes of living organisms and bird community is known to play crucial roles in the functioning of an ecosystem in which they are found. ${ }^{1,2}$ They are cosmopolitan and uneven in their distributions; their distribution, diversity and densities depend on various factors such as climate, altitude, vegetation, water availability and anthropogenic activities. ${ }^{3}$ India ranks among the top ten countries in the world in terms of the most number of bird species, ${ }^{4}$ and harbors about 1200 species of birds which makes up about $13 \%$ of the world's bird species (9600), ${ }^{5,6}$ Lepage recorded 662 species of birds in Mizoram among which 31 falls under the category of globally threatened species. ${ }^{7}$ Despite birds being the best-known class of living organisms, there are still substantial gaps in our knowledge regarding their distributions, abundances and densities. ${ }^{8}$ Studies on bird diversity are important as it raises an awareness of the need for global conservation of the avian community 9,10 and to understand the well-being of an ecosystem as a whole and help to delineate the importance of a regional or local landscape for avian conservation. Though Mizoram lies within the Indo-Myanmar biodiversity hotspot, studies on the avian community are scanty. Although there are a few existing studies that deal with pheasants and birds of a particular group and a specific area, ${ }^{11-17}$ more studies are needed to be done in order to show the picture of the Mizoram avian community. 


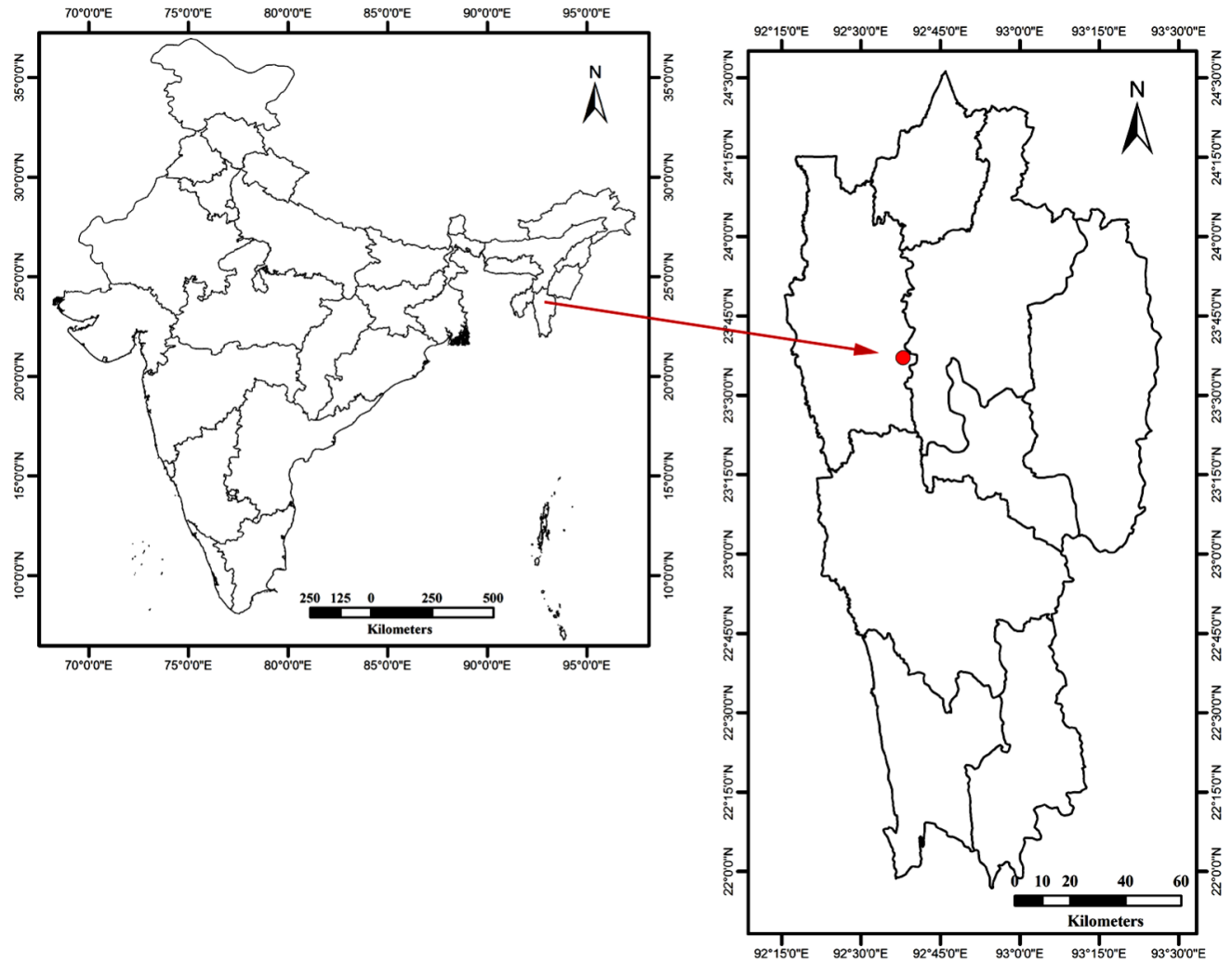

Figure 1 | Map showing study site and location of Reiek Biodiversity Spot.

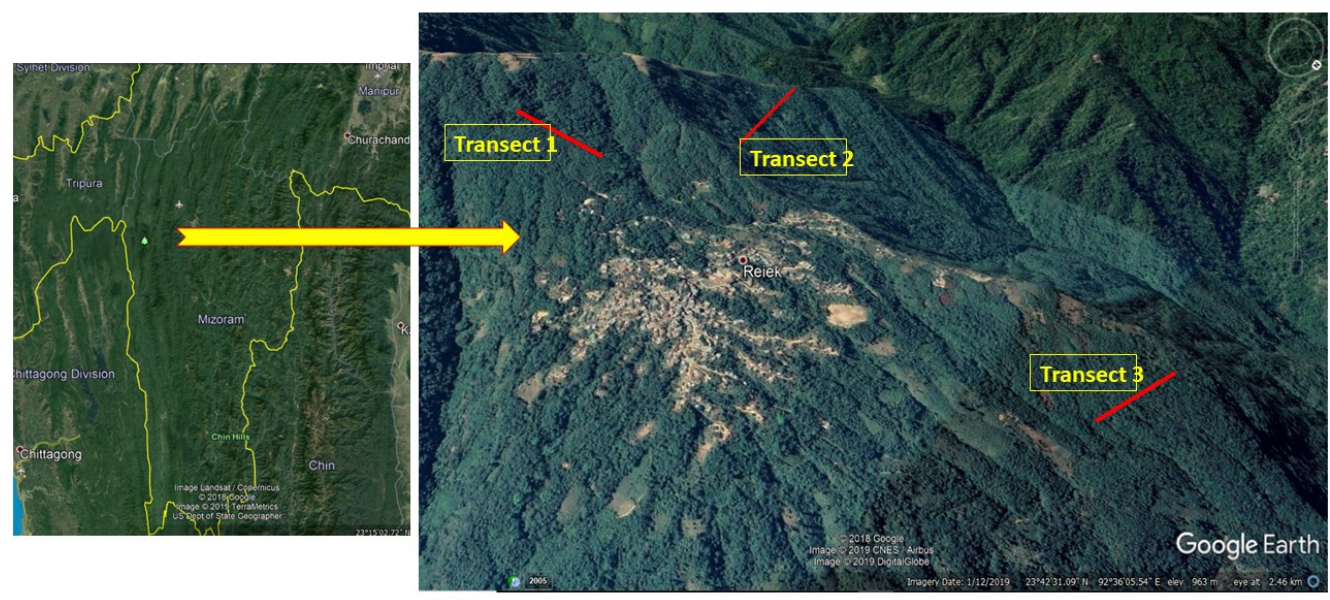

Figure 2 | Map showing study site and location of transects.

Keeping in view of the above points, the present study was carried out to add baseline information on avian species diversity and abundance in Reiek Biodiversity Spot, Mizoram, northeastern India and to create awareness for their conservation and help in strategic conservation planning. Considering the scarcity of information on the avian diversity as discussed above, the present study is designed to assess the species composition and to investigate the diversity and relative abundance of birds in Reiek Biodiversity Spot, Mizoram, India.

\section{Materials and Methods}

\section{Study site}

Reiek is a village located at $23^{\circ} 41^{\prime} 17.36^{\prime \prime} \mathrm{N}$ longitude and $92^{\circ} 36^{\prime} 07.79^{\prime \prime} \mathrm{E}$ latitude, Mizoram, northeastern India and lies within the Indo-Myanmar Biodiversity hotspot (Figures $1 \& 2$ ). It rests at an elevation of $1,325 \mathrm{~m}$ above mean sea level. The village is located at a distance of $28 \mathrm{~km}$ from the state capital, Aizawl. The area is covered by semievergreen, bamboo forest, and grasses. The major 
plant species of the area are Castanopsis tribuloides, Schima wallichii, Atalancia simplifolia and different species of bamboos and orchids.

Some portions of the community forest and its fauna are protected and conserved by the community, with the community heads and members of various non-government organizations within the village playing a vital role in this effort. Despite their efforts, tribal hunting practices using traps, snares, guns and slingshots are still prevalent among some groups of people within the community. Felling of trees and collection of firewood are also still widely prevalent in the village. Recently, the area was declared as a 'Biodiversity Spot' by the village in collaboration with Association for Environment Protection (ASEP), one of the leading NGOs in Mizoram for protecting and conserving the environment, in an effort to protect and preserve the pristine state of the protected area and its surrounding.

\section{Sampling method}

Line Transect method was employed for counting and evaluating the abundance and diversity of birds in the study. ${ }^{8}$ The study was conducted from January to March 2019. A total of three line transects were laid and followed for recording the presence of birds, viz. transect-1, transect-2 and transect-3 respectively. Transects were of different lengths and were laid on existing path, transect- 1 was laid as long as $2 \mathrm{~km}$ passing through the core of the protected area at a geological point of $23^{\circ} 41^{\prime} 23.32^{\prime \prime}$ $\mathrm{N}$ and $92^{\circ} 36^{\prime} 19.22^{\prime \prime} \mathrm{E}$, at an altitude of $1290 \mathrm{~m}$. Transect-2 was laid as long as $3.5 \mathrm{~km}$, at a geological point of $23^{\circ} 41^{\prime} 29.72^{\prime \prime} \mathrm{N}$ and $92^{\circ} 35^{\prime} 52.61^{\prime \prime} \mathrm{E}$ at an altitude of $1077 \mathrm{~m}$ above sea level. Transect-3 covered a distance of $2 \mathrm{~km}$, located at $23^{\circ} 42^{\prime} 31.09^{\prime \prime}$ $\mathrm{N}$ and $92^{\circ} 36^{\prime} 05.54^{\prime \prime} \mathrm{E}$, at an altitude of $963 \mathrm{~m}$ above mean sea level. Transect-3 was laid in a relatively disturbed area where agricultural practices and firewood collection are carried out.

Transects were walked in the morning (0530$0830 \mathrm{hrs})$ and evening (1400-1700 hrs) at an interval of six days for a period of three months. For each transect, we recorded the bird species and numbers encountered in the area. For the survey, SLR camera (Nikon P900) was used for photography, binocular (Nikon Sporter EX) and the calls of birds were used for observations, recording and identification. For identification and field diagnosis of birds, Grimmet et $a .^{17}$ and Grewal et $a l^{18}{ }^{18}$ were consulted. For diversity study, in addition to the line transect method, simple bird watching (opportunistic sampling) within the study was also adopted.

\section{Data analysis}

PAST (PAleontological STatistics) version 1.93 was used for estimating abundance and diversity indices. The relative abundance of a species was calculated by dividing the abundance of a species by the total abundance of all species combined. ${ }^{8}$

Bird diversity was calculated using both ShannonWiener and Simpson's diversity indices. ShannonWiener diversity Index was calculated using the formula:

$$
\mathrm{H}^{\prime}=-\sum_{i=1}^{R} p_{i} \ln p_{i}
$$

Where $p_{i}=$ proportion of individual species and $R=$ total number of species of the community (numbers seen and heard)

Simpson's diversity index, D was calculated using the formula:

$$
D=\frac{\sum n_{i}\left(n_{i}-1\right)}{N(N-1)}
$$

Where $n_{i}=$ the total no. of birds of each individual species and $\mathrm{N}=$ the total number of birds of all species

The value of $D$ ranges between 0 and 1 . With this index, 1 represents infinite diversity and 0 , no diversity.

\section{Result and Discussion}

\section{Species composition}

A total of 117 bird species, belonging to 37 families and 10 orders were recorded from Reiek Biodiversity Spot (Table 1). Altogether, 110 species were recorded from the transects, while the remaining 7 species were recorded outside the transects through opportunistic sampling. This record is fairly high despite the fact that the study site is being dominated by tribal communities who are known to indulge in various hunting practices and secondly, the site not being under the categories of protected area notified by the government. The relatively high avian species composition could be attributed to the availability of heterogeneous habitat, i.e., primary and secondary forests, grasses, bamboo forest, jhum land ${ }^{15}$ and secondly to the conservation efforts of the community where the communal forest is protected and conserved providing home to large number of avifauna. The bird species composition in the present study is lesser than studies that have been carried out in various protected areas around the state such as- a study carried out by Sailo and Lalthanzara in Lengteng Wildlife Sanctuary recorded 126 species of birds belonging to 35 families, ${ }^{15}$ while the famous Murlen Wildlife Sanctuary is known to harbor more than 150 species of birds. ${ }^{16}$

Another study executed by Lalthanzara and Sailo ${ }^{17}$ in Lungleng-1, a non-protected area recorded 114 species of birds belonging to 40 families, though the number of species recorded is higher in the present study, the number of the family is higher in 
Table 1 | Species composition of Reiek Biodiversity Spot (RBS).

\begin{tabular}{|c|c|c|c|c|}
\hline Order & Family & Scientific Name & Common Name & $\begin{array}{l}\text { Status } \\
\text { (IUCN) }\end{array}$ \\
\hline \multirow[t]{4}{*}{ Accipitriformes } & Accipitridae & Accipiter badius & Shikra & $\mathrm{LC}$ \\
\hline & & Accipiter virgatus & Besra & LC \\
\hline & & Pernis ptilorhycus & Oriental Honey Buzzard & LC \\
\hline & & Spilornis cheela & Crested Serpent Eagle & LC \\
\hline \multirow[t]{2}{*}{ Falconiformes } & Falconidae & Falco tinnunculus & Common Kestrel & LC \\
\hline & & Falco peregrines & Peregrine Falcon & LC \\
\hline \multirow[t]{3}{*}{ Galliformes } & Phasianidae & Arborophila rufogularis & Rufous-throated Hill Partridge & LC \\
\hline & & Bambusicola fytchii & Mountain Bamboo Partridge & LC \\
\hline & & Gallus gallus & Red Jungle Fowl & LC \\
\hline \multirow[t]{3}{*}{ Columbiformes } & Culumbidae & Streptopelia chinensis & Spotted Dove & LC \\
\hline & & Ducula aenea & Green Imperial Pegion & LC \\
\hline & & Ducula badia & Mountain Imperial Pegion & LC \\
\hline \multirow[t]{2}{*}{ Strigiformes } & Strigidae & Glaucidium cuculoides & Asian Barred Owlet & LC \\
\hline & & Strix leptogrammica & Brown Wood Owl & LC \\
\hline \multirow[t]{4}{*}{ Cuculiformes } & Cuculidae & Phaenicophaeus tristis & Green-billed Malkoha & LC \\
\hline & & Hierococcyx varius & Common hawk-Cuckoo & LC \\
\hline & & Surniculus lugubris & Square-tailed drongo Cuckoo & LC \\
\hline & & Centropus sinensis & Greater Coucal & LC \\
\hline \multirow[t]{40}{*}{ Passeriformes } & Pycnonotidae & Ixos mcclellandii & Mountain Bulbul & LC \\
\hline & & Pycnonotus cafer & Red-Vented Bulbul & LC \\
\hline & & Pycnonotus flavescens & Flavescent Bulkbul & LC \\
\hline & & Alophoixus flaveolus & White-Throated Bulbul & LC \\
\hline & & Hypsipetes leucocephalus & Black Bulbul & $\mathrm{LC}$ \\
\hline & & Hemixos flavala & Ashy Bulbul & LC \\
\hline & & Pycnonotus flaviventris & Black-crested Bulbul & LC \\
\hline & Dicaeidae & Dicaeum ignipectus & Fire-breasted Flowerpecker & LC \\
\hline & Zosteropidae & Zosterops palpebrosus & Oriental White-eye & LC \\
\hline & Dicruridae & Dicrurus remifer & Lesser Racket-tailed Drongo & LC \\
\hline & & Dicrurus paradiseus & Greater Racket-tailed Drongo & $\mathrm{LC}$ \\
\hline & & Dicrurus macrocercus & Black Drongo & $\mathrm{LC}$ \\
\hline & & Dicrurus aeneus & Bronzed Drongo & $\mathrm{LC}$ \\
\hline & & Dicrurus leucophaeus & Ashy Drongo & LC \\
\hline & Timaliidae & Garrulax leucolophus & White-crested Laughingthrush & LC \\
\hline & & Stachyris nigriceps & Grey-throated Babbler & LC \\
\hline & & Garrulax pectoralis & Greater Necklaced-laughingthrush & LC \\
\hline & & Staphida castaneiceps & Striated Yuhina & $\mathrm{LC}$ \\
\hline & & Timalia pileata & Chesnut-capped Babbler & $\mathrm{LC}$ \\
\hline & & Yuhina nigrimenta & Black-chinned Yuhina & LC \\
\hline & & Stachyris ruficeps & Rufous-capped Babbler & $\mathrm{LC}$ \\
\hline & & Pomatorhinus ferruginosus & Coral-billed scimatar Babbler & LC \\
\hline & & Pellorneum ruficeps & Puff-throated Babbler & LC \\
\hline & & Napothera brevicaudata & Streaked Wren Babbler & $\mathrm{LC}$ \\
\hline & Muscicapidae & Enicurus schistaceus & Slaty-backed Forktail & $\mathrm{LC}$ \\
\hline & & Anthipes monileger & White-gorgeted Flycatcher & LC \\
\hline & & Saxicola ferreus & Grey Bushchat & LC \\
\hline & & Phoenicurus frontalis & Blue-fronted Redstart & $\mathrm{LC}$ \\
\hline & & Enicurus leschenaultia & White-crowned Forktail & LC \\
\hline & & Cyornis unicolor & Pale-blue Flycatcher & LC \\
\hline & & Muscicapa dauurica & Asian Brown Flycatcher & LC \\
\hline & & Niltava vivida & Vivet Niltava & LC \\
\hline & & Niltava grandis & Large Niltava & LC \\
\hline & & Niltava macgrigoriae & Small Niltava & LC \\
\hline & & Tarsiger rufilatus & Himalayan Blue Tail & LC \\
\hline & & Culicicapa ceylonensis & Grey-headed Canary Flycatcher & LC \\
\hline & & Niltava sundara & Rufous-bellied Niltava & LC \\
\hline & & Copsychus malabaricus & White-rumped Shama & $\mathrm{LC}$ \\
\hline & & Ficedula albicilla & Taiga Flycatcher & LC \\
\hline & & Ficedula hodgsoni & Slaty-backed Flycatcher & LC \\
\hline
\end{tabular}




\begin{tabular}{|c|c|c|c|c|}
\hline & \multirow[t]{2}{*}{ Nectariniidae } & Aethopyga gouldiae & Mrs. Gould's Sunbird & LC \\
\hline & & Aethopyga siparaja & Crimson Sunbird & LC \\
\hline & & Aethopyga saturate & Black-throated Sunbird & LC \\
\hline & & Aethopyga ignicauda & Fire-tailed Sunbird & LC \\
\hline & & Arachnothera magna & Streaked Spiderhunter & LC \\
\hline & & Arachnothera longirostra & Little Spiderhunter & LC \\
\hline & Corvidae & Cissa chinensis & Common Green Magpie & LC \\
\hline & & Corvus macrorhynchos & Jungle Crow & LC \\
\hline & & Dendrocitta formosae & Grey Treepie & LC \\
\hline & & Dendrocitta vagabunda & Rufous Treepie & LC \\
\hline & Turdidae & Zoothera dixoni & Long-tailed Thrush & LC \\
\hline & & Myophonus caeruleus & Blue-whistling Thrush & LC \\
\hline & & Zoothera dauma & Scaly Thrush & LC \\
\hline & & Turdus obscures & Eye-browed Thrush & LC \\
\hline & Campephagidae & Pericrocotus flammeus & Scarlet Minivet & LC \\
\hline & & Coracina melaschistos & Black-winged Cuckooshrike & LC \\
\hline & & Pericrocotus cinnamomeus & Small Minivet & LC \\
\hline & & Tephrodornis virgatus & Large Woodshrike & LC \\
\hline & & Tephrodornis pondicerianus & Common Woodshrike & LC \\
\hline & Chloropseidae & Choloropsis hardwickii & Orange-bellied Leafbird & LC \\
\hline & & Chloropsis cochinchinensis & Blue-winged Leafbird & LC \\
\hline & & Chloropsis aurifrons & Gold-fronted Leafbird & LC \\
\hline & Monarchidae & Hypothymis azurea & Black-naped Monarch & LC \\
\hline & Oriolidae & Oriolus chinensis & Black-naped Oriole & LC \\
\hline & Emberizidae & Emberiza pusilla & Little Bunting & LC \\
\hline & & Emberiza rutila & Chesnut Bunting & LC \\
\hline & Cisticolidae & Orthotomus sutorius & Common tailorbird & LC \\
\hline & & Orthotomus cuculatus & Mountain tailorbird & LC \\
\hline & & Prinia atrogularis & Black-throated Prinia & LC \\
\hline & & Prinia crinigera & Striated Prinia & LC \\
\hline & & Prinia rufescens & Rufescent Prinia & LC \\
\hline & Aegithinidae & Aegithina tiphia & Common lora & LC \\
\hline & Sittidae & Sitta castanea & Chesnut-bellied Nuthatch & LC \\
\hline & & Sitta frontalis & Velvet-fronted Nuthatch & LC \\
\hline & & Sitta hmialayensis & White-tailed Nuthatch & LC \\
\hline & Eurylaimidae & Psarisomus dalhousiae & Long-tailed broadbill & LC \\
\hline & Rhipiduridae & Rhipidura albicollis & White- throated Fantail & LC \\
\hline & Vangidae & Hemipus picatus & Bar-winged flycatcher Shrike & LC \\
\hline & Motacillidae & Anthus hodgsoni & Olive-backed Pipit & LC \\
\hline & Sylviidae & Phylloscopus xanthoschistos & Grey-hooded Warbler & LC \\
\hline & & Alcippe nipalensis & Nepal Fulvetta & LC \\
\hline & & Phylloscopus inornatus & Yellow Browed Warbler & LC \\
\hline & & Phylloscopus trochiloides & Greenish Warbler & LC \\
\hline & & Phylloscopus whistleri & Whisler's Warbler & LC \\
\hline & & Seicercus poliogenys & Grey-cheeked Warbler & LC \\
\hline & Laniidae & Lanius schach & Long-tailed Shrike & LC \\
\hline & Passeridae & Passer montanus & Eurasian Tree Sparrow & LC \\
\hline & Fringillidae & Carpodacus erythrinus & Common Rosefinch & LC \\
\hline & Paridae & Melanochlora sultanea & Sultan Tit & LC \\
\hline & Vireonidae & Erpornis zantholeuca & White bellied Erpornis & LC \\
\hline Piciformes & Capitonidae & Megalaima asiatica & Blue-throated Barbet & LC \\
\hline & & Megalaima virens & Great Barbet & LC \\
\hline & & Megalaima haemacephala & Coppersmith Barbet & LC \\
\hline & Picidae & Picus flavinucha & Greater Yellownaped Woodpecker & LC \\
\hline & & Sasia ochracea & White-browed Piculet & LC \\
\hline & & Picumnus innominatus & Speckled Piculet & LC \\
\hline & & $\begin{array}{l}\text { Dendrocopos nanus } \\
\text { Blythipicus pyrrhotis }\end{array}$ & $\begin{array}{l}\text { Brown-capped Pygmy Woodpecker } \\
\text { Bay-Woodpecker }\end{array}$ & $\begin{array}{l}\mathrm{LC} \\
\mathrm{LC}\end{array}$ \\
\hline Apodiformes & Apodidae & Apus acuticauda & Dark-rumped Swift & VU \\
\hline Coraciiformes & Upupidae & Upupa epops & Common Hoopoe & LC \\
\hline
\end{tabular}


Lungleng-1 community forest as compared to Reiek biodiversity Spot. A study executed by Syiem et al. ${ }^{20}$ at Nongkhyllem landscape in Meghalaya which includes Nongkhyllem Wildlife Sanctuary and its surrounding matrix recorded a total of 94 species which is lesser than species recorded from the present study site.

Family-wise species composition indicates that Muscicapidae has the largest family comprising of 16 species, followed by Timaliidae comprising of 10 species, and Pycnonotidae with 7 species, Nectariniidae with 6 species. 5 families, viz. Dicruridae, Campephagidae, Cistocolidae, Sylviidae and Picidae were represented by 5 species. Accipitridae, Cuculidae, Turdidae and Corvidae were represented by 4 species each. Chloropsidae, Sittidae, Capatonidae, were represented by 3 species each. Strigidae and Emberizidae consist of 2 species each. 17 families were represented by single species (Figure 3). The recorded highest species richness in Muscicapidae family is in accordance with records from various parts of Mizoram, ${ }^{17,21}$ as well as the entire Northeast region ${ }^{22}$. This could be due to their ability to adapt to different habitats including human -modified habitats. Meanwhile, it has been reported that Timaliidae family has the greatest number of species in Lengteng Wildlife Sanctuary, Mizoram in a study executed by Sailo and Lalthanzara ${ }^{15}$. Saikia and Saikia also reported that Sylvidae has the largest family in northeast India represented by 153 species and 44 genera. ${ }^{23}$

As expected, Passeriformes being the most diverse group of birds, $^{24}$ order-wise species composition revealed that Passeriformes has the highest species composition with 89 species recorded under this order, followed by Paciformes, comprising of 8 species, Accipitriformes and Cuculiformes came third in the list with 4 species each (Figure 4).

The family Passeriformes was represented by 27 families, topping the list of orders having the highest number of families, followed by Piciformes represented by 2 families. The remaining 8 orders were represented by 1 family each (Figure 5 ).

A total of 8 raptor species were recorded while 3 ground bird species and a species belonging to the most aerial birds of all families, i.e. Dark-rumped Swift were recorded from the present study. Of all the birds recorded, only Dark-rumped Swift (Apus acuticauda) falls under the Vulnerable category of the IUCN Red List of Threatened Species (2018), ${ }^{25}$ while the remaining 116 species belong to the Least Concern category.

Most of the species recorded from Reiek Biodiversity Spot belong to the Least Concerned category of the IUCN Red List of Threatened Species could probably be due to the fact that the area is under constant interaction with human, and that species under Threatened, Near Threatened, Vulnerable and other special categories tend to avoid humans and are usually specialist species requiring certain conditions to thrive well in a place. ${ }^{17}$

\section{Species diversity}

The Shannon-Wiener diversity for Reiek Biodiversity Spot was calculated to be $\mathrm{H}^{\prime}=3.858$. This record is fairly high and indicates that the area has great potential for avian conservation site, dominance $D=0.04146$ and Simpson Diversity was calculated to be 0.9585 . Reiek landscape, providing heterogeneous habitats could be the reason for the relatively high diversity index.

The result of the diversity index revealed that Transect-3 has the highest Shannon-Wiener diversity index, followed by Transect-1 and Transect-2 respectively (Table 2). Even though Transect-3 was laid in a fairly disturbed area where shifting cultivation site and secondary forest from shifting cultivation occurs, it recorded the highest ShannonWiener Diversity index as compared to the two transects. This could be attributed to the area is a mixture of secondary forest and agricultural land offering a more heterogeneous habitat than the other two transects, hence it allows various species of birds to co-exist ${ }^{26}$ and bird species are easier to

Table 2 | Transect-wise diversity indices.

\begin{tabular}{lccc}
\hline & Transect-1 & Transect-2 & Transect-3 \\
\hline Taxa & 63 & 52 & 61 \\
Individuals & 263 & 297 & 219 \\
Dominance & 0.07811 & 0.07726 & 0.04072 \\
Shannon,H' & 3.339 & 3.167 & 3.622 \\
Simpson_1-D & 0.9219 & 0.9593 & 0.9593 \\
Evenness_e^H/S & 0.4473 & 0.4564 & 0.612 \\
\hline
\end{tabular}

Table 3 | Family-wise diversity indices.

\begin{tabular}{lccc}
\hline Family & Domi-nance & Shannon, H' & $\begin{array}{c}\text { Simpson_1- } \\
\text { D }\end{array}$ \\
\hline Accitripidae & 0.3333 & 1.242 & 0.6667 \\
Strigidae & 0.5556 & 0.6365 & 0.4444 \\
Phasianidae & 0.4489 & 0.8823 & 0.5511 \\
\hline Culumbidae & 0.4286 & 0.9557 & 0.5714 \\
\hline Cuculidae & 0.5 & 0.8676 & 0.5 \\
\hline Pycnonotidae & 0.1948 & 1.774 & 0.8052 \\
Dicruridae & 0.2676 & 1.445 & 0.6779 \\
\hline Timaliidae & 0.3221 & 1.475 & 0.6779 \\
\hline Muscicapidae & 0.08587 & 2.598 & 0.9141 \\
\hline Nectariniidae & 0.3979 & 1.203 & 0.6021 \\
Corvidae & 0.3086 & 1.273 & 0.6914 \\
\hline Turdidae & 0.46 & 0.8979 & 0.54 \\
\hline Campephagidae & 0.6966 & 0.6751 & 0.3034 \\
Chloropseidae & 0.4063 & 0.9743 & 0.5938 \\
\hline Emberizidae & 0.625 & 0.5623 & 0.375 \\
\hline Cisticolidae & 0.24 & 1.505 & 0.76 \\
\hline Sittidae & 0.375 & 1.04 & 0.625 \\
\hline Sylviidae & 0.7106 & 0.6571 & 0.2694 \\
Capatonidae & 0.4897 & 0.8 & 0.5103 \\
\hline Picidae & 0.2711 & 1.415 & 0.7289 \\
\hline & & &
\end{tabular}


Figure 3 | Family-wise species composition.

Figure 4 | Order-wise species composition.

Figure $\mathbf{5}$ | Number of families belonging to different orders.

Figure 6 | Family-wise relative abundance.
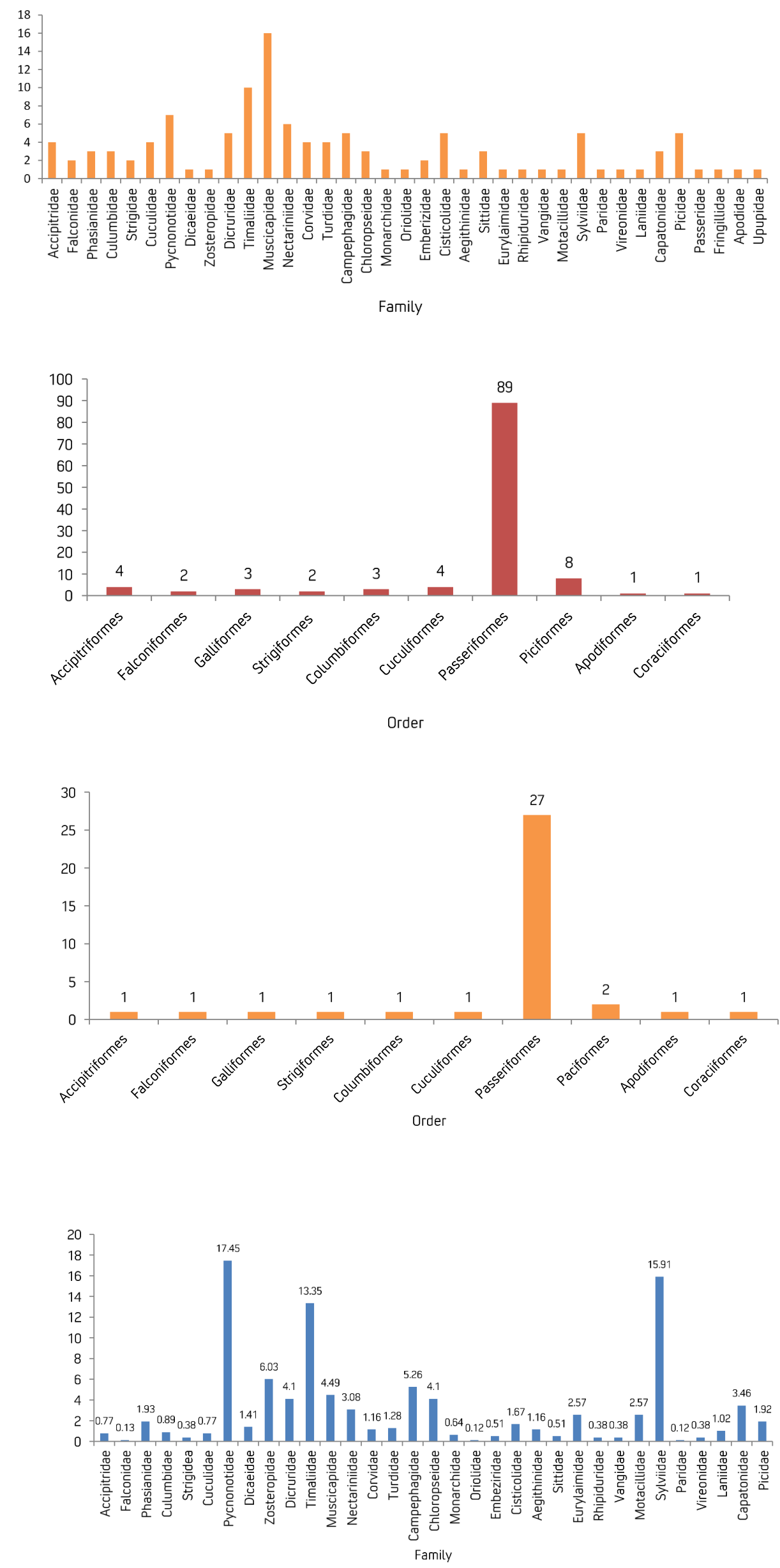
Table 4 | Relative abundance of species.

\begin{tabular}{|c|c|c|c|}
\hline SI No & Scientific Name & Common name & $\begin{array}{c}\text { Relative } \\
\text { abundance \% }\end{array}$ \\
\hline 1 & Accipiter badius & Shikra & 0.39 \\
\hline 2 & Pernis ptilorhycus & Oriental Honey Buzzard & 0.13 \\
\hline 3 & Spilornis cheela & Crested Serpent Eagle & 0.13 \\
\hline 4 & Accipiter virgatus & Besra & 0.13 \\
\hline 5 & Falco peregrines & Peregrine Falcon & 0.13 \\
\hline 6 & Arborophila rufogularis & Rufous-throated Hill Partridge & 1.03 \\
\hline 7 & Bambusicola fytchii & Mountain Bamboo Partridge & 0.77 \\
\hline 8 & Gallus gallus & Red-Jungle Fowl & 0.13 \\
\hline 9 & Streptopelia chinensis & Spotted Dove & 0.51 \\
\hline 10 & Ducula aenea & Green Imperial Pegion & 0.13 \\
\hline 11 & Ducula badia & Mountain Imperial Pegion & 0.26 \\
\hline 12 & Glaucidium cuculoides & Asian Barred Owlet & 0.26 \\
\hline 13 & Strix leptogrammica & Brown Wood Owl & 0.13 \\
\hline 14 & Phaenico phaeustristis & Green-billed Malkoha & 0.51 \\
\hline 15 & Hierococcyx varius & Common hawk-Cuckoo & 0.13 \\
\hline 16 & Surniculus lugubris & Square-tailed Drongo Cuckoo & 0.13 \\
\hline 17 & Ixos mcclellandi & Mountain Bulbul & 1.41 \\
\hline 18 & Pycnonotus cafer & Red-Vented Bulbul & 5.13 \\
\hline 19 & Pycnonotus flavescens & Flavescent Bulbul & 1.54 \\
\hline 20 & Alophoixus flaveolus & White-throated Bulbul & 1.93 \\
\hline 21 & Hypsipetes leucocephalus & Black Bulbul & 2.95 \\
\hline 22 & Hemixos flavala & Ashy Bulbul & 1.54 \\
\hline 23 & Pycnonotus flaviventris & Black-crested Bulbul & 2.95 \\
\hline 24 & Dicaeum ignipectus & Fire-breasted Flowerpecker & 1.41 \\
\hline 25 & Zosterops palpebrosus & OrientalWhite-eye & 6.03 \\
\hline 26 & Dicrurus remifer & Lesser Racket-tailed Drongo & 0.51 \\
\hline 27 & Dicrurus paradiseus & Greater Racket-tailed Drongo & 0.77 \\
\hline 28 & Dicrurus macrocercus & Black Drongo & 1.67 \\
\hline 29 & Dicrurus aeneus & Bronzed Drongo & 0.90 \\
\hline 30 & Dicrurus leucophaeus & Ashy Drongo & 0.26 \\
\hline 31 & Garrulax leucolophus & White-crested Laughingthrush & 0.51 \\
\hline 32 & Stachyris nigriceps & Grey-throated Babbler & 0.26 \\
\hline 33 & Garrulax pectoralis & Greater Necklaced-laughingthrush & 1.67 \\
\hline 34 & Staphida castaniceps & Striated Yuhina & 8.60 \\
\hline 35 & Timalia pileata & Chesnut-capped Babbler & 0.39 \\
\hline 36 & Yuhina nigrimenta & Black-chinned Yuhina & 0.51 \\
\hline 37 & Stachyris ruficeps & Rufous-capped Babbler & 0.13 \\
\hline 38 & Pomatorhinus ferruginosus & Coral-billed scimatar Babbler & 1.03 \\
\hline 39 & Pellorneum ruficeps & Puff-throated Babbler & 0.13 \\
\hline 40 & Napothera brevicaudata & Streak wren Babbler & 0.13 \\
\hline 41 & Enicurus schistaceus & Slaty-backed Forktail & 0.51 \\
\hline 42 & Anthipes monileger & White-gorgeted Flycatcher & 0.13 \\
\hline 43 & Saxicola ferreus & Grey Bushchat & 0.13 \\
\hline 44 & Phoenicurus frontalis & Blue-fronted Redstart & 0.13 \\
\hline 45 & Cyornis unicolor & Pale-blue Flycatcher & 0.13 \\
\hline 46 & Muscicapa dauurica & Asian Brown Flaycatcher & 0.26 \\
\hline 47 & Niltava vivida & VivetNiltava & 0.26 \\
\hline 48 & Niltava grandis & Large Niltava & 0.26 \\
\hline 49 & Niltava macgrigoriae & Small Niltava & 0.51 \\
\hline 50 & Tarsiger rufilatus & Himalayan Blue Tailed & 0.77 \\
\hline 51 & Culicicapa ceylonensis & Grey headedcanary Flycatcher & 0.39 \\
\hline 52 & Niltava sundara & Rufous-bellied Niltava & 0.13 \\
\hline 53 & Copsychus malabaricus & White-rumpedShama & 0.26 \\
\hline 54 & Megalaima asiatica & Blue-throated Barbet & 1.28 \\
\hline 55 & Megalaima virens & Great Barbet & 2.05 \\
\hline 56 & Megalaima haemacephala & Coppersmith Barbet & 0.13 \\
\hline 57 & Picus flavinucha & Greater Yellownaped & 0.64 \\
\hline 58 & Sasia ochracea & White-browed Piculet & 0.13 \\
\hline 59 & Picumnus innominatus & Speckled Piculet & 0.64 \\
\hline
\end{tabular}




\begin{tabular}{|c|c|c|c|}
\hline 60 & Dendrocopos nanus & Brown-capped Pygmy Woodpecker & 0.13 \\
\hline 61 & Blythipicus pyrrhotis & Bay-Woodpecker & 0.39 \\
\hline 62 & Aethopyga gouldiae & Mrs. Gould's Sunbird & 0.26 \\
\hline 63 & Aethopyga siparaja & Crimson Sunbird & 0.13 \\
\hline 64 & Aethopyga saturate & Black-throated Sunbird & 0.39 \\
\hline 65 & Aethopyga ignicauda & Fire-tailed Sunbird & 0.13 \\
\hline 66 & Arachnothera magna & Streaked Spiderhunter & 1.28 \\
\hline 67 & Cissa chinensis & Common Green Magpie & 0.26 \\
\hline 68 & Corvus macrorhynchos & Jungle Crow & 0.13 \\
\hline 69 & Zoothera dixoni & Long-tailed Thrush & 0.13 \\
\hline 70 & Myophonus caeruleus & Blue-whisling Thrush & 0.77 \\
\hline 71 & Zoothera dauma & Scaly Thrush & 0.39 \\
\hline 72 & Pericrocotus flammeus & Scarlet Minivet & 4.36 \\
\hline 73 & Coracina melaschistos & Black-winged Cuckooshrike & 0.13 \\
\hline 74 & Pericrocotu cinnamomeus & Small Minivet & 0.26 \\
\hline 75 & Choloropsis hardwickii & Orange-bellied Leafbird & 2.05 \\
\hline 76 & Chloropsis cochinchinensis & Blue-winged Leafbird & 0.51 \\
\hline 77 & Chloropsis aurifrons & Gold-fronted Leafbird & 1.54 \\
\hline 78 & Hypothymis azurea & Black-naped Monarch & 0.64 \\
\hline 79 & Oriolus chinensis & Black-naped Oriole & 0.13 \\
\hline 80 & Orthotomus sutorius & Common Tailorbird & 0.39 \\
\hline 81 & Orthotomus cuculatus & Mountain Tailorbird & 0.26 \\
\hline 82 & Prinia atrogularis & Black-throated Prinia & 0.77 \\
\hline 83 & Emberiza pusilla & Little Bunting & 0.39 \\
\hline 84 & Emberiza rutila & Chesnut Bunting & 0.13 \\
\hline 85 & Aegithina tiphia & Common lora & 1.16 \\
\hline 86 & Sitta castanea & Chesnut-bellied Nuthatch & 0.13 \\
\hline 87 & Sitta frontalis & Velvet-fronted Nuthatch & 0.13 \\
\hline 88 & Psarisomus dalhousiae & Long-tailed Broadbill & 2.57 \\
\hline 89 & Rhipidura albicollis & White-throated Fantail & 0.39 \\
\hline 90 & Tephrodornis pondicerianus & Common Woodshrike & 0.39 \\
\hline 91 & Hemipus picatus & Bar-winged Flycatcher Shrike & 0.39 \\
\hline 92 & Anthus hodgsoni & Olive-backed Pipit & 2.57 \\
\hline 93 & Phylloscopus xanthoschistos & Grey-hooded Warbler & 0.90 \\
\hline 94 & Alcippe nipalensis & Nepal Fulvetta & 13.35 \\
\hline 95 & Lanius schach & Long-tailed Shrike & 1.03 \\
\hline 96 & Arachnothera longirostra & Little Spiderhunter & 0.90 \\
\hline 97 & Dendrocitta formosae & Grey Treepie & 0.51 \\
\hline 98 & Erpornis zantholeuca & White-bellied Erponis & 0.39 \\
\hline 99 & Seicercus poliogenys & Grey-Cheeked Warbler & 0.13 \\
\hline 100 & Phylloscopus whistleri & Whisler Warbler & 0.39 \\
\hline 101 & Ficedula hodgsoni & Slaty-backed Flycatcher & 0.39 \\
\hline 102 & Melanochlora sultanea & Sultan Tit & 0.13 \\
\hline 103 & Ficedula albicilla & Taiga Flycatcher & 0.26 \\
\hline 104 & Dendrocitta vagabunda & Rufous Treepie & 0.26 \\
\hline 105 & Phylloscopus inornatus & Yellow-browed Warbler & 0.77 \\
\hline 106 & Prinia rufescens & Rufescent Prinia & 0.13 \\
\hline 107 & Prinia crinigera & Striated Prinia & 0.13 \\
\hline 108 & Sitta hmialayensis & White-tailed Nuthatch & 0.26 \\
\hline 109 & Tephrodornis virgatus & Large Woodshrike & 0.13 \\
\hline 110 & Phylloscopus trochiloides & Greenish Warbler & 0.39 \\
\hline
\end{tabular}

detect for the observer owing to the less dense characteristics. But this cannot rule out the fact that an intact, undisturbed area usually host specialist, important and conservation-worthy species, ${ }^{27}$ as such Dark-rumped Swift and important ground birds such as Rufous-throated Hill Partridge, Mountain Bamboo Partridge and Red-jungle Fowl, in this case, were recorded from transect- 1 and transect- 2 within the protected site. This finding is parallel to the work of Syiem et al. ${ }^{20}$ in Ri Bhoi district of Meghalaya, India where non-protected areas have higher diversity than protected areas, however, the protected area supports more special, conservationworthy species. Raman et al. ${ }^{28}$ in their study of avifauna in Dampa landscape, Mizoram also revealed that secondary forests clearly play the role of important habitat for birds.

Among the families of birds observed, 
Muscicapidae has the highest Shannon- Wiener diversity index, $\mathrm{H}^{\prime}=2.598$, followed by Pycnonotidae with an index of $\mathrm{H}^{\prime}=1.774$ and Cisticolidae with an index of $\mathrm{H}^{\prime}=1.505$ (Table 3).

\section{Relative abundance}

In terms of relative abundance, Nepal Fulvetta (Alcippe nipalensis) has the highest relative abundance (13.35\%) among all the species observed, which is followed by Striated Yuhina (Staphida castaniceps) with relative abundance of $8.6007 \%$ and Oriental White-eye (Zosterops palpebrosus) with relative abundance of $6.03337 \%$ (Table 4). Nepal Fulvetta ( $A$. nipalensis) being the highest in terms of relative abundance could be attributed to their feeding habit, preference of habitat and behavior and the same applies for birds that came second and third in the list, Striated Yuhina and Oriental Whiteeye. ${ }^{2}$ These birds are highly gregarious and forage in groups, and this gives them the advantages of foraging success, selection of mate, reduced predation and spotting of forage location. ${ }^{29}$ Their relatively gregarious behavior also probably gives the observer an increased chance of sighting and recording them.

Of all the families recorded, Pycnonotidae has the highest relative abundance $(17.45 \%)$, followed by Sylviidae $15.91 \%$ and Timaliidae $13.35 \%$ (Figure 6). Pycnonotidae, topping the list in terms of relative abundance could be probably due to the generalist characteristic of this family enabling them to adapt to various types of habitat, feeding on a wide variety of fruits and arthropods. ${ }^{30}$ Some Bulbuls are known to have a high tolerance to disturbance, ${ }^{31}$ Redvented Bulbul, Black-crested Bulbul were recorded from all the three transects.

\section{Conclusion}

117 species of birds belonging to 37 families and 10 orders were recorded from Reiek Biodiversity Spot, and the Shannon-Wiener Diversity index was calculated to be $H^{\prime}=3.85$. Family-wise species composition shows that Muscicapidae has the largest family comprising of 16 species, followed by Timaliidae with 6 species. Among all the species recorded, Alcippe nipalensishas the highest relative abundance (13.35\%), followed by Staphida castaniceps (Striated Yuhina) and Zosterops palpebrosus (Oriental White Eye) with $8.6007 \%$ and $6.03337 \%$ each. Family-wise relative abundance revealed that Pycnonotidae has the highest relative abundance (17.45\%), followed by Sylviidae (15.91\%) and Timaliidae (13,35\%). Among the species recorded Apus acuticauda (Dark-rumped Swift) belongs to the Vulnerable Category of the IUCN Red List Category for Threatened Species while the remaining 116 belong to Least Concern category.

This shows that the area supports a rich and diverse bird community in spite of it being an area which is not notified as a protected site by the State Government and has a good potential for avian conservation site and for bird watching tourism as well. This high diversity could be attributed to the heterogeneous habitat of the area and the conservation effort of the people. The present study also revealed that the secondary forest and cultivation site supports a good diversity of birds. The distribution of birds across the landscape and their association with their habitats were not studied due to time and financial constraint, hence, further studies on these regards are recommended.

Better management of the community protected site along with the adjacent secondary forest and cultivation site is recommended. Conservation awareness among the communities should be enhanced especially among the hunters of the community.

\section{Acknowledgement}

We are grateful to Mr. C Lalthakima, Reiek, an avian expert and guide for his help during the field study, and Association for Environment Protection (ASEP) and Reiek Village Community for their cooperation and necessary permission.

\section{Authors' contributions}

H. Lalthanzara and S.S. Sundaravel designed the study; Lalruatkimi and L. Sailo collected the data; Betsy Zodinpuii, L. Sailo and Lalruatkimi analysed the data; Lalruatkimi, S.S. Sundaravel and H. Lalthanzara prepared the manuscript.

\section{Conflict of interest}

The authors declare no conflict of interest.

\section{References}

1. Ramachandra, A.M. (2013). Diversity and richness of bird species in newly formed habitats of Chandoli National Park in Western Ghats, Maharashtra State, India. Biodiversity Journal, 4(1), 235-242.

2. Girma, Z., Mengesha, G., Asfaw, T. (2017). Diversity, relative abundance and distribution of avian fauna in and around Wondo Genet Forest, South-Central Ethiopia. Research Journal of Forestry, 11(1), 1-12.

3. Rodriguez-Estrella, R. (2007). Land use changes affect distributional pattern of desert birds in Baja California Peninsula, Mexico. Diversity and Distribution, 13, 877-889.

4. Praveen, J., Jayapal, R., Pittie, A. (2016). A checklist 
of the birds of India. Indian Birds, 11(5), 113.

5. Sethy, J., Samal, D., Sethi, S., Baral, B., Jena, S., Payra, S., Das, N.G., Boruah, B., Sahu, K.H. (2015). Species diversity and abundance of birds in and around North Orissa University, Takatpur, Baripade, Mayurbanj, Odisha. International Journal of Innovative Research in Science, Engineering and Technology, 4(2), 301.

6. Ali, S., Ripley, S.D. (1987). Handbook of the Birds of India and Pakistan. New Delhi. Oxford Press.

7. Lepage, D. (2017). Avibase - Bird Checklist of the World, Mizoram. https://avibase.bsc-eoc.org/ checklist.jsp?region=INnemz\&list=clements March 2019)

8. Bibby, C., Jones, M., Marsden, S. (2000). Bird Surveys, Expedition Field Technique. Cambridge. Birdlife International.

9. Sondhi, N.S. (2002). A comparison of birds community of two fragmented and two continuous South East Asia forest. Biodiversity and Conservation, 11, 1105-1119.

10. Voon, M.F.A., Nasradhi, N.K., Rahman, A.M., Azlan, M.J. (2014). Bird diversity, density and foraging activities in a university campus landscape in Sarawak. Borneo Journal of Resource Science and Technology, 20(9), 9-20.

11. Lalthanzara, H. (2010). Recent status of threatened birds of Mizoram. Science Vision, 10 (4), 168-169.

12. Lalthanzara, H., Vanramliana, Lalramliana. (2011). Pheasants of Mizoram (India): Present status of diversity and distribution. Science Vision, 11(4), 218 -223 .

13. Kasambe, R., Lalthanzara, H., Lalawmawia Sailo (2013). Bird survey in Lengteng Wildlife Sanctuary and Blue Mountain (Phawngpui) National Park, Mizoram. BUCEROS, 18(3), 6-13.

14. Lalthanzara, H., Sailo, L., Solanki, G.S., Ramanujam, S.N. (2014). Galliformes and their conservation issues in Mizoram, India. Cibtech Journal of Zoology, 3(1), 42.

15. Sailo, L., Lalthanzara, H. (2015). Bird diversity of Lengteng Wildlife Sanctuary, Mizoram. Science Vision, 15(2), 87-96.

16. Anonymous. (2015). Important Bird Area factsheet: Murlen National Park. http:// www.birdlife.org. (13 March 2019)

17. Lalthanzara, H., Sailo L. (2017). Avian diversity in Lungleng-1, Mizoram. International Journal of Recent Scientific Research, 8(6), 18033-18038.

18. Grimmett, R., Inskipp, C., Inskipp, T. (2011). Birds of the Indian Subcontinent. London. Christopher
Heler, an imprint of Bloomsbury Publishing.

19. Grewal, B. (2000). Birds of the Indian Subcontinent. Hongkong. Local Column Limited.

20. Syiem, L.N., Goswami, B., Vasudev, D. (2018). In a Tree by the Brook, there's a song bird who sings: Woodlands in an agricultural matrix maintain functionality of a wintering bird community. PLOS One, 13(8), e0201657.

21. Vanlalsawmi, R., Solanki, G.S., Zakhuma (2011). Birds of Dampa Tiger Reserve in Mizoram, India. Proceedings of Advances in Environmental Chemistry, 242-244.

22. Narwade, S., Kalra, M., Jagdish, R., Vavier, D., Satpute, S., Khan, N., Talukdar, G., Mathur, B.V., Vasudevan, K., Pundir, S.D., Chavan, V., Sood, R. (2002). Literature based species occurrence data of birds of North East India. Zookeys, 150, 140-417.

23. Saikia, K.P., Saikia, K.M. (2000). Diversity of bird fauna in North East India. Journal of the Assam Science Society, 41(4), 379-396.

24. Raikow, J.R., Bledsoc, H.A. (2000). Phylogeny and evolution of the passerine birds. BioScience. 50(6), $487-499$

25. IUCN (2018). IUCN Red List of Threatened Species. Version 2018.2. https:// www.iucnredlist.org/ . (16 March 2019.)

26. Casas, G., Darski, B., Ferreira, A.M.P., Kindel, A., Muller, C. (2016). Habitat structure influence the diversity, richness and composition of bird assemblages in successional Atlantic Rainforest. Tropical Conservation Science, 9(1), 503-524.

27. Darras, K., Rahman, D., Sugito, W., Prawiradila, D., Agus, R., Fitriwan, I., Tscharntke, T. Birds of primary and secondary forest and shrub habitats in the peat swamp of Berbak National Park, Sumatra. F1000 Research, 7, 229.

28. Raman, S.R,K., Rawat, S.G., Johnsingh, T.J.A. (1998). Recovery of tropical rainforest avifauna in relation to vegetaion succession following shifting cultivation in Mizoram, North East, India. Journal of Applied Ecology, 35, 214-231

29. Kwok, K.H. (2017). Flocking behavior of forest birds in HongKong. Journal of Forest Research, 28(5), 1097-1101.

30. Shakya, B.S., Sheldon, H.F. (2017). The phylogeny of the world's bulbuls (Pycnonotidae) inferred using a supermatrix approach. Ibis 157,498-509.

31. Corlett, R.T. (1998). Fruigivory and seed dispersal by vertebrates in the Oriental (IndoMalayan) region. Biological Reviews. 73, 413-448. DOI: $10.1017 /$ s0006323198005234 